Cataract

\section{Tackling the greatest challenge in cataract surgery}

\section{F Chang}

\section{From the standpoint of cost effectiveness manual small incision cataract surgery is clearly superior to the alternatives}

D espite all that modern technology has done to advance the treatment of cataracts, our greatest challenge continues to be the large and increasing backlog of cataract blindness in developing countries. ${ }^{1-5}$ While in North America and western Europe, intraocular lens (IOL) research and development are primarily directed towards reversing lens ageing (presbyopia), millions in developing nations with reversible blindness caused by cataracts go untreated.

Modern phacoemulsification machines are expensive to purchase and maintain, have relatively high disposable costs, and require extensive surgical training. Furthermore, for the more advanced and mature cataracts typical of underserved populations, performing phacoemulsification becomes more difficult and complication prone. What is needed is a high volume, cost effective, low technology procedure that can treat the most advanced of cataracts with a low complication rate in the shortest amount of time.

This very goal is being achieved in a handful of international programmes that are providing a hopeful paradigm for overcoming worldwide cataract blindness. I have had the privilege of visiting both the Aravind Eye Hospital network in southern India, and the Tilganga Eye Centre in Kathmandu, Nepal. Seeing first hand how their systems provide high volume, low cost cataract surgery is an awe inspiring experience for any visiting ophthalmologist.

Founded in 1976 by the now 87 year old Dr G Venkataswamy, Aravind Eye Hospital has grown into a network of five regional eye hospitals providing high level ophthalmic care to the poor population of southern India. Private paying patients comprise approximately $30 \%$ of their patient base. This revenue funds $70 \%$ of their services that are provided at no cost to the indigent via a financially self sustaining programme that receives minimal government reimbursement. In terms of cataract surgery, this means that of the approximately
200000 procedures performed annually in the Aravind system, $70 \%$ are provided free.

While private cataract patients at Aravind may pay anywhere from \$200$\$ 300$ to undergo phacoemulsification with foldable IOLs imported from the United States, the non-paying cataract patients are treated for less than $\$ 15$ per case, including the IOL. This is accomplished by performing a manual, sutureless, small incision extracapsular procedure with re-usable equipment and supplies. ${ }^{6-9}$ Their IOL manufacturing facility, Aurolab, produces poly(methlymethacrylate) (PMMA) IOLs for less than $\$ 5$ per lens. Following a retrobulbar block, the nucleus is expressed through a capsulorhexis and a temporal, self sealing $6.0-6.5 \mathrm{~mm}$ scleral pocket incision. Manual cortical cleanup precedes capsular bag implantation of a PMMA IOL. The technique is commonly abbreviated as manual SICS (small incision cataract surgery).

An efficient, high volume system utilising low cost, sub- 5 minute procedures to tackle advanced cataracts with minimal complications is clearly the best way to use the scarcest and most precious asset of the system - the cataract surgeon

While the procedure itself seems straightforward, it is the stunning speed, skill, and efficiency with which it is performed that must literally be seen to be believed. By alternating between two parallel operating room tables, a single surgeon is able to perform over 15 cases per hour by consistently completing sub-5 minute procedures on the densest of cataracts with no intervening turnover time. To ensure efficiency across different surgical teams, every aspect of the procedure is standardised, from preoperative patient and instrument preparation to the surgical steps themselves. Having been screened in outlying eye camps, as many as $300-400$ cataract patients will by bussed to an Aravind eye hospital where they will all undergo their surgery on a single day. After several days of in-house follow up, they are transported back to their rural villages where a local postoperative visit and refraction are performed 1 month later by the Aravind staff. This standardised Aravind system streamlines and centralises cataract care by performing all surgery in the main hospital. ${ }^{10}$

Founded in 1994 by Dr Sanduk Ruit, the Tilganga Eye Centre is a shining example of an efficient eye care delivery system on a smaller scale. Dr Ruit has developed his own variation of the manual, sutureless SICS. ${ }^{11-13}$ Tilganga Eye Centre is also financially self sustaining wherein private care subsidises charity care. They also have their own IOL manufacturing facility, which, like that at Aravind, is able to supply low cost IOLs to other developing countries. Because the rural population in Nepal is so widely scattered among mountain villages that are accessible only by foot, the Tilganga system strives to deliver portable cataract care by transporting the necessary staff and equipment to remote eye camps. ${ }^{14}{ }^{15}$ Using a single portable operating table, the Tilganga surgeons can also perform more than 10 cataract surgeries per hour. As at Aravind, the high volume, cost effective Tilganga surgical techniques and protocols are standardised across their surgical teams.

Though of a different scale and serving different types of communities, Aravind and Tilganga are complementary models of how best to address the world's backlog of cataract blindness. They demonstrate that the solution requires not just a cost effective surgical technique, but also an entire system of efficient and financially self sustaining cataract care delivery. There must be a system for attracting, screening, diagnosing, and transporting cataract patients to and from rural camps. There must be a source of low cost IOLs, medications, and supplies. Most importantly, there must be highly coordinated teams of dedicated ophthalmologists and support staff, who execute their roles with military precision. To assure maximum efficiency and reproducibility, there must be a standard protocol for every aspect of care.

In this issue of BJO (p 1079), Venkatesh and co-authors provide a detailed outcome study of the high volume, manual SICS (small incision cataract surgery) method used at Aravind. Two days' surgical volume for three cataract surgeons ( $\sim 600$ cases) were randomly selected and reviewed retrospectively. Despite a high percentage of advanced and mature cataracts, operative complications were extremely 
low, and vitreous loss occurred in less than $1 \%$ of cases. Understandably, stringent postoperative outcome data with long term follow up are not easily attainable in a rural cataract camp population. However, with 6 week follow up of nearly $90 \%$ of the patients, 95\% achieved a best corrected visual acuity of at least 6/18 (not excluding macular or other pathology). These outcomes are all the more impressive considering that almost $90 \%$ of the patients had preoperative vision of 5/60 or worse, and that the surgical time for these nearly 600 patients averaged 3.75 minutes.

How does the manual, sutureless SICS compare to other cataract methods? One prospective randomised study determined that this technique resulted in better uncorrected acuity than standard extracapsular cataract extraction. ${ }^{16}$ Astigmatism control is particularly important in populations that have limited access to spectacles. I recently participated in a prospective randomised trial at the Tilganga Eye Centre comparing manual, sutureless SICS with phaco in a cataract camp population. While the data analysis is not complete, I can attest to the difficulty of performing phaco in a camp setting with a high incidence of advanced cataracts and poor corneal visibility. Finally, from the standpoint of cost effectiveness, manual SICS is clearly superior to the alternatives. ${ }^{17} 18$

Outcome studies such as these provide convincing evidence that surgical systems, such as those at Aravind and
Tilganga, are the most promising, efficacious, and cost effective means to eradicate cataract blindness in developing countries. Beyond the impressive productivity of these two institutions, equally important has been their desire and ability to train surgical teams from other developing countries in their methods of cataract surgery. An efficient, high volume system utilising low cost, sub-5 minute procedures to tackle advanced cataracts with minimal complications is clearly the best way to use the scarcest and most precious asset of the system-the cataract surgeon.

Br J Ophthalmol 2005;89:1073-1074.

doi: $10.1136 /$ bjo.2005.068213

Correspondence to: D F Chang, University of California, San Francisco, Los Áltos, CA 94024, USA; dceye@earthlink.net

\section{REFERENCES}

1 World Health Organization. Global initiative for the elimination of avoidable blindness, WHO Fact Sheet No 213. Geneva: WHO, Feb, 2000.

2 Dandona R, Dandona L. Socioeconomic status and blindness. $\mathrm{Br} J$ Ophthalmol 2001;85:1484-8.

3 Arnold J. Global cataract blindness: the unmet challenge [editorial]. Br J Ophthalmol 1998:82:593-4.

4 Thylefors B. A global initiative for the elimination of avoidable blindness [editorial]. Am J Ophthalmol 1998;125:90-3.

5 Minassian DC, Mehra V. 3. 8 million blinded by cataract each year: Projections from the first epidemiological study of incidence of cataract blindness in India, $\mathrm{Br} J$ Ophthalmol 1990;74:341-3.

6 Civerchia L, Ravindran RD, Apoorvananda SW, et al. High volume intraocular lens surgery in a rural eye camp in India. Ophthalmic Surg Lasers 1996;27:200-8
7 Prajna NV, Chandrakanth Ks, Kim R, et al. The Madurai intraocular lens study II: clinical outcomes. Am J Ophthalmol 1998;125:14-25.

8 Natchiar G, DabralKar T. Manual small incision suture less cataract surgery-An alternative technique to instrumental phacoemulsification. Operative Techniques Cataract Refract Surg 2000;3:161-70.

9 Balent LC, Narendran K, Patel S, et al. High volume sutureless intraocular lens surgery in a rural eye camp in India. Ophthalmic Surg Lasers 2001;32:446-55.

10 Natchiar G, Robin AL, Thulasirai R, et al. Attacking the backlog of India's curable blind; the Aravind Eye Hospital model. Arch Ophthalmol 1994; 112:987-93.

11 Ruit S, Tabin GC, Nissman SA, et al. Low-cost high-volume extracapsular cataract extraction with posterior chamber intraocular lens implantation in Nepal. Ophthalmology 1999;106: 1887-92.

12 Ruit S, Paudyal G, Gurung R, et al. An innovation in developing world cataract surgery: sutureless extracapsular cataract extraction with intraocular lens implantation. Clin Experiment Ophthalmol 2000;28:274-9.

13 Hennig A, Kumar J, Yorston D, et al. Sutureless cataract surgery with nucleus extraction: outcome of a prospective study in Nepal. Br J Ophthalmol 2003;87:266-70.

14 Pokharel GP, Regmi G, Shrestha SK, et al. Prevalence of blindness and cataract surgery in Nepal. Br J Ophthalmol 1998:82:600-5.

15 Pokharel GP, Selvaraj S, Ellwein LB. Visual functioning and quality of life outcomes among cataract operated and unoperated blind populations in Nepal. Br J Ophthalmol 1998:82:606-10.

16 Gogate PM, Deshpande M, Wormald RP, et al Extracapsular cataract surgery compared with manual small incision cataract surgery in community eye care setting in western India: a randomised controlled trial. Br J Ophthalmol 2003;87:667-72.

17 Gogate PM, Deshpande M, Wormald RP. Is manual small incision cataract surgery affordable in developing countires? A cost comparison with extracapsular cataract extraction. Br J Ophthalmol 2003;87:843-6.

18 Muralikrishnan R, Venkatesh R, Prajna NV, et al. Economic cost of cataract surgery procedures in an established eye care centre in Southern India. Ophthalmic Epidemiol 2004;11:369-80.

\section{Periorbital haemangiomas}

\section{W V Good}

\section{Paediatric ophthalmology's stepchild?}

E very paediatric ophthalmology practice cares for infants and young children with periorbital haemangioma, and yet there is very little evidence based research to guide treatment. Options for management generally include topical, intralesional or systemic steroids, surgical resection, or no treatment at all, as haemangioma usually involute after a period of growth. When tumours block the visual axis, aggressive intervention is mandatory. In almost every other case, management is based on anecdote and clinical judgment and, in many cases, is optional or elective.

The aetiology of periocular haemangioma is also debated. Current opinion holds that haemangiomas occur more frequently in premature infants, yet older research on this subject suggests that this finding is caused by ascertainment bias-that is, premature infants are observed more often than full term infants, leading observers to conclude that premature infants develop haemangioma more commonly. ${ }^{12}$ Why do haemangioma have a predilection for areas around the eyes, or do they? The astigmatism seen with periocular haemangioma is usually attributed to pressure effect of the tumour, but is there evidence to support this conclusion, or to validate that this is always the cause of astigmatism? Why does astigmatism often persist after the tumour regresses? Which locations for tumours are most likely to cause or contribute to amblyopia? Again, the answer to these questions is not fully known.

Such is the state of the practice of medicine as it pertains to these tumours: many questions with far too few answers. And so it is ironic and fitting that Ranschod and colleagues in their paper reported in this issue of the $B J O$ ( $\mathrm{p}$ 1134) shed no light on management of periorbital haemangioma, despite a thorough review of the literature and a thoughtful meta-analysis of research on the subject. Rather than 
criticise the authors for taking readers of the BJO down a blind alley, we should be grateful to them for identifying a very important and generally disregarded problem, the periorbital haemangioma. While this tumour continues to occur at a significant frequency, and to threaten vision and appearance, its epidemiology, cause, biology, and management are in need of further study.

Should the reader then conclude that the periocular haemangioma is, indeed, a sort of stepchild in paediatric ophthalmology research, largely ignored and second fiddle to other conditions such as amblyopia and strabismus? If the answer to this is affirmative, then it may be useful to explore the means to beat this condition. One reason might be that the pathology and effects of these tumours generally fall outside the purview of many of us. The periorbital haemangioma, after all, is a tumour, often responsible for cosmetic changes. Ophthalmologists generally do not have experience with laser treatments for haemangioma, and may be happy enough to defer systemic treatment implementation to paediatricians and dermatologists. Consider, too, that very serious side effects from ophthalmologist led treatment have been reported, including blindness associated with intralesional injection.

\section{While this tumour continues to occur at a significant frequency, and to threaten vision and appearance, its epidemiology, cause, biology, and management are in need of further study.}

I believe that a different reason exists for the relative scientific neglect of haemangioma and for the low number of controlled clinical trials on this subject. The tumour is protean, its biology and growth variable, and its location around the eyes highly variable in terms of extent, location, and size. These facts make it very difficult to compare treatments at all. How can treatment of a globular haemangioma of the upper outer lid be compared to the same treatment for the same type and size tumour of the upper inner lid? Never mind that the haemangioma could be diffuse, affect both lids, start at an earlier age in some infants, occur in a premature infant, or occur in conjunction with tumours in other locations. All of these factors could influence the effect of the haemangioma on refractive error, amblyopia, tumour growth, and so on. Scientists have understandably avoided controlled studies on this problem, because experimental design is difficult.

Consequently, case reports have provided the foundation for knowledge about periocular haemangioma and their management. Case reports and series are often maligned as second class science, anecdotal, uncontrolled, and failing to test a hypothesis. But without case reports noting that astigmatism sometimes improves with intralesional injections, or of side effects of treatment, including adrenal suppression and blindness, clinicians would have very little data at all to guide management of haemangioma. Case reports will continue to be important to describe effects of treatment for this condition.

Lastly, we should try to imagine the design of a controlled clinical trial to test treatment for periorbital haemangioma. This is the charge set forth by Ranshod, and it is not an easy one. Controlling time of onset of haemangioma, size, location, degree of astigmatism at outset, premature status, and presence or absence of intermittent occlusion are just a few of the issues. All of these variables could be controlled in a large enough trial with, perhaps, a photographic reading centre where masked investigators would interpret external findings.

The recent explosion of developmental and molecular biology research offers an intriguing and different venue for research on this subject, too. The haemangioma's biological behaviour dictates its effects on vision. This is obvious in the case of occlusion. The large and rapid growing tumour is more likely to cause occlusion, amblyopia, and astigmatism, whether or not one ascribes to the pressure theory of haemangioma induced astigmatism. Tumour biology could influence astigmatism and cosmesis in more subtle ways, as well. Knowledge that a given haemangioma has biological characteristics linked to rapid growth, or astigmatism, or significant cosmetic changes, would help guide clinical intervention. An amalgamation of clinical and basic science research for the study of management of periorbital haemangioma could lead to earlier detection of problematic tumours.

Let's hope that Ranshod and colleagues, and others, carry forth their concerns about this neglected problem and work to rectify the current situation, where a shortage of evidence is used to support clinical decision making. The periorbital haemangioma deserves full citizenship in the world of paediatric ophthalmology research.

Br J Ophthalmol 2005;89:1074-1075.

doi: 10.1136/bjo.2005.073411

Correspondence to: W V Good, Smith Kettlewell Eye Research Institute, 2318 Fillmore Street, San Francisco, CA 94115, USA; Good@Ski.org

\section{REFERENCES}

1 Silverman WA. Missing and unaccounted for. Paediatr Perinat Epidemiol 2004;18:95-6.

2 Holmdahl K. Cutaneous hemangiomas in premature and mature infants. Acta Paediatr 1955;44:370-4. 
Childhood myopia

\section{Monovision slows myopia progression}

\section{J A Guggenheim, C H To}

\section{Increased chances of finding an effective optical method of arresting myopia development}

l:

The Marriage of Heaven and Hell, William Blake says that "If the doors of perception were cleansed everything would appear to man as it is: infinite." In vision, of course, there is a simple connection between optical infinity and perceived visual clarity, at least for distance vision in emmetropes. Contact lens practitioners and refractive surgeons have taken things one step further. By exploiting the brain's ability to perceptually suppress central vision in one eye when the two eyes are receiving disparate stimuli, they have found that it is often possible to correct presbyopic ametropes using a distance correction for the dominant eye, and a near correction for the non-dominant eye. In this "monovision" situation, patients thus have to suppress the central vision in their non-dominant eye for distance tasks, and in their dominant eye for near tasks.

In essence, monovision is a form of deliberately introduced anisometropia, and it is this property that John Phillips has exploited in a highly original study, in this issue of BJO ( $p$ 1196), that provides new insight into the role of blur in regulating eye growth and refractive development in children. In this small clinical trial, children received a full myopic correction for their dominant eye and an undercorrection of up to $+2.00 \mathrm{D}$ for their non-dominant eye (as discussed below, the undercorrection led to the vision in the children's non-dominant eyes being continually blurred). The results were striking: the rate of myopia progression in the undercorrected eye was found to be approximately $50 \%$ of that in the fully corrected eye. Furthermore, the reduced rate of myopic progression was attributed to a reduced rate of vitreous chamber elongation, consistent with a slowing of the primary structural change responsible for causing myopia.

Using the chicken as a model, Schaeffel and co-workers ${ }^{1}$ first showed that refractive state could be altered in response to retinal blur imposed by wearing a spectacle lens over one or both eyes. Remarkably, the change in refractive error Schaeffel et al found depended on the sign of the defocus: negative spectacle lenses, which would have diverged the light entering the eye and shifted the image plane behind the retina (leading to "hyperopic defocus") induced myopia. Meanwhile, positive spectacle lenses, which would have converged the light entering the eye, shifting the image plane in front of the retina (producing "myopic defocus") induced hyperopia. In each case, the refractive change was mostly the result of an altered rate of vitreous chamber elongation. Chicks becoming myopic showed an acceleration in their normal rate of axial eye growth. Chicks developing hyperopia showed a slowing or cessation of the normal rate. Similarly elegant experiments have demonstrated that refractive plasticity of this kind, which represents an active form of emmetropisation, is a feature of the early development of many other species, including marmosets ${ }^{2}$ and monkeys. $^{3}$

So it seems highly likely that human infants also use blur cues to guide refractive development during emmetropisation. What is less clear is whether blur contributes to the development of myopia in school aged children, as well. A decade ago, Gwiazda and colleagues ${ }^{4}$ hypothesised that the lag of accommodation at near could explain the intriguing link between near work and myopia. Children with an accommodative lag at near would experience hyperopic blur at the retina that, according to the animal models, would lead to myopia development. However, conclusive proof for this theory is lacking, and more recent results from a longitudinal study suggest that there is no difference in accommodative lag before the onset of myopia development, between those children who remain emmetropic and those who become myopic. ${ }^{5}$

Furthermore, experiments in which animals have been allowed short periods (minutes) of unrestricted vision, in between long periods of hyperopic defocus, show that these intervals of sharp focus quickly counteract the tendency towards myopia. This might mean that children undergoing even prolonged periods of near work induced blur are protected from myopia by distance viewing in between times. Also, Chung and colleagues ${ }^{6}$ undercorrected both eyes of a group of 47 young myopes, by approximately $+0.75 \mathrm{D}$. After 2 years, instead of the myopic blur these children having been exposed to making them less myopic, Chung et al found that the children had become even more myopic than a control group who had been fully corrected. So perhaps humans are unusual, in not being able to emmetropise in response to defocus? Or perhaps by the time they reach school age, the plasticity of the human emmetropisation system has decreased below a clinically significant level?

Even before the study by Phillips, however, there was indirect evidence that exposure to blur could influence refractive development in school age children. In the COMET study, a randomised, multicentre, double masked clinical trial to evaluate whether progressive addition lenses (PALs) slowed myopia development in children, PALs were indeed found to slow myopia progression significantly, though only by about $14 \%$ over 3 years. While it is possible that reduced accommodation itself was responsible for this slowed rate of progression, it seems more likely to have been the reduction in hyperopic defocus brought about by the extra plus power at near lessening the stimulus for myopia development, since in the subgroup of children who had larger lags of accommodation, PALs slowed myopia progression to a greater extent (by $21 \%$ in general, and by $37 \%$ in children who also had esophoria at near). ${ }^{7}$

How does the monovision study carried out by Phillips add to our knowledge? Crucially, unlike presbyopic monovision wearers, the children in the Phillips study were found to posture their accommodation according to their dominant eye, for both distance and near. This meant that the non-dominant eye would have experienced myopic blur in both viewing conditions, and hence throughout the period of spectacle wear. However, the amount of accommodation these children exerted would presumably have been similar to that when wearing a full distance correction. Therefore, the new study strongly suggests that it was the continuous myopic blur experienced by the undercorrected eye that signalled its slower rate of myopic progression.

Critics might argue that the study by Phillips is too small to get overly excited about; after all only 13 children were involved in the trial. Yet the "within subject" design afforded by the monovision approach (compared to the "between subject" design necessitated by myopia control trials such as the COMET study) meant that despite its 
small size, the study was adequately powered, as the results confirm. However, the lack of a control group does leave open room for a slight doubt: because we do not know what the baseline rate of myopia progression is in this particular group of children, it is conceivable that the fully corrected eyes had shown an accelerated rate of myopia progression, rather than the undercorrected eyes having shown a slower rate of progression.

The new study immediately brings to mind two key questions. Firstly, is it possible to exploit this monovision paradigm clinically to slow myopia progression? And, secondly, why did the undercorrected eyes still progress towards myopia, instead of halting their progression completely?

In answer to the first question, it is important to emphasise that the protocol used by Phillips induced anisometropia, because only the non-dominant eye was exposed to myopic blur. Therefore, clinically, some kind of periodic reversal of the treatment regimen would be required, so that the dominant eye also spent time exposed to myopic blur while the non-dominant eye received clear vision. A crucial issue is whether children will tolerate the undercorrection of their dominant eyes in the reversed monovision situation. If they do, then the prospects look promising, but much work will still be required to optimise the treatment. For instance, would greater amounts of blur give a stronger effect, or would this tip the balance and induce form deprivation myopia? What would be the optimal treatment duration-for example, would it be better to switch the undercorrection on alternate days, alternate weeks, or alternate months, perhaps? Also, a more rigorous study design, including masked observers and a fully corrected control group will be required to prove conclusively that monovision is effective. It should noted, as well, that if a $50 \%$ reduction in myopia progression turns out to be the best that can be achieved, then taking into account the fact that the undercorrection would only be present for $50 \%$ of the time, then the overall progression rate might be slowed by only $25 \%$, which would be disappointing.

Why should the undercorrected eyes have become more myopic, despite the myopic blur they were exposed to? At this age, the eye is normally elongating at approximately $0.2 \mathrm{~mm}$ per year, ${ }^{8}$ so there may be an endogenous, developmental push towards axial elongation. However, in animal models, visual cues are generally able to dominate this innate propensity, to effectively halt axial elongation completely. Table 1 of Phillips's paper shows that there was some intersubject variation in response, and an impression that full time monovision wearers may have derived a slightly greater benefit than part-time wearers. If so, then at least part of the explanation may be that during periods when the monovision correction was not worn, the non-dominant eyes experienced hyperopic defocus (either because of children reverting to a conventional spectacle correction, or doing near work while uncorrected). Alternatively, there may be additional environmental risk factors for myopia progression ${ }^{9}$ to which these children were exposed, or it could be that once myopia progression has begun, that it is somehow self perpetuating (there is a precedent for this in marmosets ${ }^{10}$ ).

In conclusion, by providing evidence that further implicates blur in driving the progression of myopia in school age children and, most importantly, in providing arguably the strongest evidence yet that myopia progression can be slowed by imposing myopic blur at the retina, this study makes the chances of finding an effective optical method of arresting myopia development look significantly brighter.

Br J Ophthalmol 2005;89:1076-1077. doi: $10.1136 /$ bjo.2005.068106

\section{Authors' affiliations}

J A Guggenheim, School of Optometry and Vision Sciences, Cardiff University, King

Edward VII Avenue, Cardiff CF10 3NB, UK

C H To, Department of Optometry and Radiography, Hong Kong Polytechnic

University, Hung Hom, Hong Kong

Correspondence to: Jez Guggenheim, Cardiff University, King Edward VII Avenue, Cardiff CF10 3NB, UK; Guggenheim@cardiff.ac.uk

Competing interests: none declared

\section{REFERENCES}

1 Schaeffel F, Glasser A, Howland HC Accommodation, refractive error and eye growth in chickens. Vis Res 1988;28:639-57.

2 Graham B, Judge SJ. The effects of spectacle wear in infancy on eye growth and refractive error in the marmoset (Callithrix jacchus). Vis Res 1999;39: 189-206

3 Hung L-F, Crawford MU, Smith EL III. Spectacle lenses alter eye growth and the refractive status of young monkeys. Nat Med 1995;1:761-5.

4 Gwiazda J, Thorn F, Baver J, et al. Myopic children show insufficient accommodative response to blur. Invest Ophthalmol Vis Sci 1993:34:690-4

5 Mutti DO, Mitchell GL, Jones LA, et al. Accommodative lag at the onset of myopia in children. Invest Ophthalmol Vis Sci 2004;45:E3514

6 Chung K, Mohidin N, O'Leary DJ. Undercorrection of myopia enhances rather than inhibits myopia progression. Vis Res 2002;42:2555-9.

7 Gwiazda JE, Hyman L, Norton TT, et al. Accommodation and related risk factors associated with myopia progression and their interaction with treatment in COMET children. Invest Ophthalmol Vis Sci 2004;45:2143-51.

8 Larsen JS. The sagittal growth of the eye. IV. Ultrasonic measurement of the axial length of the eye from birth to puberty. Acta Ophthalmol 1971:49:873-86.

9 Saw S-M, Katz J, Schein OD, et al. Epidemiology of myopia. Epidemiol Rev 1996;18:175-87.

10 Troilo D, Judge SJ. Ocular development and visual deprivation myopia in the common marmoset (Callithrix jacchus). Vis Res 1993;33:1311-24. 